\title{
CHAOS AND DYNAMICAL
}

SYSTEMS 


\section{PRIMERS IN COMPLEX SYSTEMS}

\section{Editorial Advisory Board}

John H. Miller, Editor-in-Chief, Carnegie Mellon University and Santa Fe Institute

Murray Gell-Mann, Santa Fe Institute

David Krakauer, Santa Fe Institute

Simon Levin, Princeton University

Mark Newman, University of Michigan

Dan Rockmore, Dartmouth College

Geoffrey West, Santa Fe Institute

Jon Wilkins, Santa Fe Institute

\section{Volumes Published in the Series}

Chaos and Dynamical Systems

by David P. Feldman (2019)

Viruses as Complex Adaptive Systems, by Ricard Solé and Santiago F. Elena (2019)

Natural Complexity: A Modeling Handbook,

by Paul Charbonneau (2017)

Spin Glasses and Complexity,

by Daniel L. Stein and Charles M. Newman (2013)

Diversity and Complexity,

by Scott E. Page (2011)

Phase Transitions,

by Ricard V. Solé (2011)

Ant Encounters: Interaction Networks and Colony Behavior, by Deborah M. Gordon (2010) 


\title{
CHAOS AND
}

\section{DYNAMICAL}

\section{SYSTEMS}

\author{
David P. Feldman
}

PRINCETON UNIVERSITY PRESS

Princeton \& Oxford 
Copyright (C) 2019 by Princeton University Press

Requests for permission to reproduce material from this work should be sent to permissions@press.princeton.edu

Published by Princeton University Press

41 William Street, Princeton, New Jersey 08540

6 Oxford Street, Woodstock, Oxfordshire OX20 1TR

press.princeton.edu

All Rights Reserved

LCCN: 2019936804

ISBN 978-0-691-16152-5

British Library Cataloging-in-Publication Data is available

Editorial: Vickie Kearn, Susannah Shoemaker, and Lauren Bucca

Production Editorial: Nathan Carr

Text Design: Pamela L. Schnitter

Jacket/Cover Design: Pamela L. Schnitter

Jacket/Cover Credit: April Vollmer, Kosha 9, 2010, woodcut and mixed media collage on panel, $12 \times 12$ inches.

Production: Erin Suydam

Publicity: Matthew Taylor and Kathryn Stevens

Copyeditor: Lor Campbell Gehret

This book has been composed in Adobe Garamond and Helvetica Neue

Printed on acid-free paper. $\infty$

Printed in the United States of America

$\begin{array}{llllllllll}1 & 3 & 5 & 7 & 9 & 10 & 8 & 6 & 4 & 2\end{array}$ 